\title{
Spectroscopic Survey of Rapidly Oscillating Ap Stars
}

\author{
W. W. Weiss ${ }^{1}$, T. A. Ryabchikova ${ }^{1,2}$, F. Kupka ${ }^{1}$, T. R. Lueftinger ${ }^{1}$, \\ I. S. Savanov ${ }^{3}$, V. P. Malanushenko ${ }^{3}$
}

\begin{abstract}
A spectroscopic survey of roAp stars has been initiated in Vienna in order to determine their fundamental astrophysical parameters and abundances. We report here on our attempt to confirm and elaborate an atmospheric peculiarity recently discovered (Ryabchikova et al. 1999) which should allow to identify roAp stars with a single spectrum.
\end{abstract}

\section{Introduction}

The mentioned criterion will have a major impact on the asteroseismology of stars in that part of the HR diagram as it would provide an efficient tool to enlarge the group of roAp stars without being dependent on excellent photometric data. We expect to gain further insights in the specific atmospheric structure of roAp stars and to answer as yet open questions on the mechanisms responsible for the oscillations, the role of the magnetic field in mode selection, diffusion and atmospheric inhomogeneities (spots).

\section{Observations}

High resolution, high signal-to-noise $\mathrm{CCD}$ and Reticon spectra have been obtained at the 3.6-m and NTT telescopes of ESO (La Silla), the $2.6-\mathrm{m}$ telescope of Crimean Astrophysical Observatory (Ukraine), the 6-m and 1-m telescopes of Special Astrophysical Observatory (Russia), and the 2.7-m telescope of McDonald Observatory.

\section{Discussion}

Abundances determined from lines of the third spectrum of various rare earth elements (Pr III and Nd III in our case, see Table 1) appear to be overabundant by about 1 dex (!) and even more relative to singly ionized spectra. The suspicion that this effect might be caused by incorrect atomic data is contradicted by the fact that a similar analysis for non-pulsating Ap stars with otherwise similar properties does not show this imbalance. Results of the abundance determinations for $\mathrm{Si}, \mathrm{Cr}, \mathrm{Fe}, \mathrm{Pr}$, and $\mathrm{Nd}$ in program stars are given in Table 1. From 2 to

\footnotetext{
${ }^{1}$ Institute for Astronomy, University of Vienna, Türkenschanzstrasse 17, A-1180 Wien, Austria

${ }^{2}$ Institute of Astronomy, RAS, 48 Pyatnitskaya Str., Moscow 109017, Russia

${ }^{3}$ Crimean Astrophys. Observatory; I. Newton. Inst. of Chile, Crimea, Ukraine
} 
10 lines of Pr III and Nd III were used to derive abundances depending on the spectral regions covered for individual stars, effective temperature, blending, etc. The observed difference might be the first solid indication of intrinsically different internal atmospheric structures of roAp relative to non-roAp stars. If so, this effect should help to constrain the presently discussed excitation mechanisms. It is unclear which physical parameters distinguish roAp from non-pulsating Ap and from 'normal' stars. For one star, $\gamma$ Equ, there is an observational evidence for the connection between the observed REE anomaly and pulsations. Savanov, Malanushenko, \& Ryabchikova (1998) found that maximum amplitude of the radial velocity variations due to pulsation is observed for Pr III and Nd III lines. It is very important to check this effect for other roAp stars.

Presently, we have made full abundance analyses of seven roAp stars with solar scaled Kurucz model atmospheres. All synthetic calculations have been made with the SYNTHMAG code (Piskunov 1999), which treats line formation in the presence of a magnetic field. For HD 42659 we derived abundances for a few of the most important elements.

Table 1. Si, Cr, Fe, Pr, and $\mathrm{Nd}$ abundances in the atmospheres of program stars with error estimates in brackets. For Pr and Nd abundances for different ions are given separately.

\begin{tabular}{|c|c|c|c|c|c|c|}
\hline \multirow[t]{2}{*}{ Star } & \multicolumn{2}{|c|}{$\log [\mathrm{Si} / \mathrm{H}] \log [\mathrm{Cr} / \mathrm{H}] \log [\mathrm{Fe} / \mathrm{H}]$} & \multicolumn{2}{|c|}{$\log [\mathrm{Pr} / \mathrm{H}]$} & \multicolumn{2}{|c|}{$\log [\mathrm{Nd} / \mathrm{H}]$} \\
\hline & & & Pr II & Pr III & $\mathrm{Nd}$ II & Nd III \\
\hline HR 4816 & -3.80 & $-3.61(10)-3.46(10)$ & $-8.70:$ & $-8.76(30)$ & $-7.90(30)$ & $-8.06(20)$ \\
\hline HR 7575 & -4.43 & $-4.21(10)-3.98(10)$ & $-9.00:$ & $-8.91(30)$ & $-8.15(30)$ & $-7.86(20)$ \\
\hline$\beta \mathrm{CrB}$ & -4.80 & $-4.56(10)-4.15(10)$ & $-9.31(20)$ & $-9.31(20)$ & $-8.61(20)$ & $-8.32(05)$ \\
\hline HD 184471 & - & $-4.56(20)-4.26(20)$ & $-10.10(40)$ & - & $-9.72(30)$ & $-9.69(25)$ \\
\hline HD 42659 & -5.30 & $-4.90(20)-4.56(30)$ & $-9.10(30)$ & $-7.84(22)$ & $-8.65(30)$ & $-7.42(21)$ \\
\hline$\alpha \operatorname{Cir}$ & -4.60 & $-5.31(25)-4.46(10)$ & $-10.36(10)$ & $-8.72(23)$ & $-9.26(25)$ & $-7.60(06)$ \\
\hline HD 166473 & -4.06 & $-5.36(30)-4.30(22)$ & $-8.77(22)$ & $-7.56(38)$ & $-7.93(28)$ & $-6.39(37)$ \\
\hline$\gamma \mathrm{Equ}$ & -4.65 & $-5.31(30)-4.31(25)$ & $-9.80(24)$ & $-8.47(05)$ & $-8.91(22)$ & $-7.46(28)$ \\
\hline $10 \mathrm{Aql}$ & -4.15 & $-5.09(17)-4.24(21)$ & $-10.13(27)$ & $-9.17(30)$ & $-9.73(29)$ & $-7.18(54)$ \\
\hline BI Mic & -4.80 & $-5.50(20)-4.46(15)$ & $-10.05(37)$ & $-8.76(23)$ & $-9.46(27)$ & $-7.54(26)$ \\
\hline DO Eri & -5.15 & $-5.81(20)-5.06(15)$ & $-9.70(30)$ & $-8.04(24)$ & $-8.56(27)$ & $-7.10(20)$ \\
\hline HD 122970 & -4.41 & $-5.95(20)-4.44(18)$ & $-10.00(21)$ & $-8.59(30)$ & $-9.19(27)$ & $-7.99(26)$ \\
\hline
\end{tabular}

Acknowledgments. This project received funding from the Austrian Fonds zur Förderung der wissenschaftlichen Forschung.

\section{References}

Piskunov, N. E. 1999, in ASSL Vol. 243, Proc. 2nd Int. Workshop on Solar Polarization, Bangalore, India 1998, ed. K. N. Nagendra \& J. O. Stenflo (Dordrecht: Kluwer), 515

Ryabchikova, T. A., Savanov, I. S., Malanushenko, V. P., \& Kudryavtsev, D. A. 1999, Astronomy Reports, in press

Savanov, I. S., Malanushenko, V. P., \& Ryabchikova, T. A. 1998, IBVS, No. 4650 